 \\ Journal of Mathematics and Computer Science
}

\section{Rough Pythagorean fuzzy ideals in ternary semigroups}

\author{
Ronnason Chinram ${ }^{\mathrm{a}, \mathrm{b}, *}$, Thammarat Panityakul ${ }^{\mathrm{a}}$ \\ ${ }^{a}$ Algebra and Applications Research Unit, Department of Mathematics and Statistics, Faculty of Science, Prince of Songkla University, \\ Hat Yai, Songkhla 90110, Thailand. \\ ${ }^{b}$ Centre of Excellence in Mathematics, CHE, Si Ayuthaya Road, Bangkok 10400, Thailand.
}

\begin{abstract}
A ternary semigroup is a nonempty set equipped with an associative ternary operation. A Pythagorean fuzzy set is one of the generalizations of the fuzzy set. The aim of this paper is to study rough Pythagorean fuzzy ideals in ternary semigroups. This idea is extended to the lower and upper approximations of Pythagorean fuzzy ideals.
\end{abstract}

Keywords: Fuzzy sets, Pythagorean fuzzy sets, rough sets, ternary semigroups.

2010 MSC: 03E72, 20N10.

(C)2020 All rights reserved.

\section{Introduction}

The notion of fuzzy sets was introduced by Zadeh [23] in 1965. Several research was conducted on the generalizations of the notion of fuzzy sets. The study of fuzzy algebraic structures started by Rosenfeld [19] in 1971. Rosenfeld introduced the notion of fuzzy groups and showed that many results in groups can be extended in an elementary manner to develop the theory of fuzzy groups. The concept of fuzzy ideals in semigroups was first developed by Kuroki (see [9, 10]). Pawlak [15] introduced the fundamental rough set concept in 1982. This concept has been developed and applied to computer science, particularly information systems. Kuroki [11] defined rough ideals in semigroups. He introduced the notion of a rough left (right, two-sided, bi-) ideal in semigroups and gave some properties of such ideals. Furthermore, the theory of rough ideals in other structures has also been studied by many authors, for example, rough ideals of $\Gamma$-semigroup studied in [2, 7, 8], Prasertpong and Siripitukdet [17] introduced a rough set in a universal set based on cores of successor classes with respect to level in a closed unit interval under a fuzzy relation, and some interesting properties were investigated, etc.. A Pythagorean fuzzy set [21, 22] is one of generalizations of the fuzzy set. After its existence, several researchers have studied. Peng and Yang presented some results on Pythagorean fuzzy sets in [16]. Garg [3, 4] proposed the weighted averaging and geometric aggregation operators using Einstein t-norm operators for solving

\footnotetext{
*Corresponding author

Email address: ronnason.c@psu.ac.th (Ronnason Chinram)

doi: $10.22436 /$ jmcs.020.04.04
}

Received: 2019-08-20 Revised: 2020-02-02 Accepted: 2020-02-04 
the decision-making problems under Pythagorean fuzzy sets environment. Naz et al. proposed a new graph by using the concept of Pythagorean fuzzy sets, called Pythagorean fuzzy graph in [14].

A ternary semigroup is a nonempty set equipped with an associative ternary operation. Every semigroup can be considered to be ternary semigroup. The existence of ternary operations originated from the study of a ternary analogue of Abelian group in 1932 by Lehmer [12]. The notion of ternary semigroups came from the problem of Banach who created an example of ternary semigroups which is not reducible to a semigroup. In addition, he conjectured that every ternary semigroup may be extended to reducible to a semigroup (cf. [13]). Los [13] exposed Banach's problem and showed that the operation in the ternary semigroup is an extension of the binary operation satisfying associative law on some nonempty set. Then many properties of ternary semigroups have been extensively studied by many authors. For example, Iampan conceptualized ideal extensions in ternary semigroups in [6], Thongkam and Changphas introduced the concept of two-sided bases of a ternary semigroup in [20], Ansari and Yaqoob [1] discussed the concept of T-rough ternary subsemigroups, T-rough ideals, T-rough bi-ideals, and T-rough interior ideals in ternary semigroups, straddles in ternary semigroups were studied in [18], etc.. The research on ternary semigroups in many aspects has been creative and interesting. It is significant to note that analogues of the results of ternary semigroups can be obtained from semigroup theory. Recently, Hussain et al. [5] presented the idea of rough Pythagorean fuzzy ideals in semigroups and discussed lower and upper approximations of Pythagorean fuzzy ideals, bi-ideals, and interior ideals in semigroups. In this paper, we introduce rough Pythagorean fuzzy ideals in ternary semigroups and give some remarkable properties.

\section{Preliminaries}

\subsection{Ternary semigroups}

Definition 2.1. A ternary semigroup is a nonempty set $T$ together with a ternary operation $(a, b, c) \mapsto[a b c]$ satisfying the associative law

$$
[[a b c] u v]=[a[b c u] v]=[a b[c u v]] \text { for all } a, b, c, u, v \in T .
$$

The following examples show that a ternary semigroup does not necessarily reduce an ordinary semigroup.

Example 2.2 (Banach's example). Let $T=\{-i, 0, i\}$ be a ternary semigroup under multiplication over complex numbers. We have that $\mathrm{T}$ is not a binary semigroup under multiplication over complex numbers.

Example 2.3. Let $\mathbb{Z}^{-}$be a ternary semigroup under multiplication over integer numbers. We have that $\mathbb{Z}^{-}$is not a binary semigroup under multiplication over integer numbers.

Let $T$ be a ternary semigroup. A function $f: T \rightarrow[0,1]$ is called a fuzzy subset of $T$.

Definition 2.4. Let $f$ and $g$ be fuzzy subsets of a ternary semigroup $T$.

(1) $f \subseteq g$ if $f(y) \leqslant g(y)$ for all $y \in T$.

(2) $(f \cap g)(y)=\min \{f(y), g(y)\}$ for all $y \in T$.

(3) $(f \cup g)(y)=\max \{f(y), g(y)\}$ for all $y \in T$.

Definition 2.5. A fuzzy subset $f$ of a ternary semigroup $T$ is called

(1) a fuzzy ternary subsemigroup of $\mathrm{T}$ if $\mathrm{f}([\mathrm{xyz}]) \geqslant \min \{f(x), f(y), f(z)\}$ for all $x, y, z \in T$;

(2) a fuzzy left ideal of $T$ if $f([x y z]) \geqslant f(z)$ for all $x, y, z \in T$;

(3) a fuzzy right ideal of $\mathrm{T}$ if $\mathrm{f}([\mathrm{xyz}]) \geqslant \mathrm{f}(\mathrm{x})$ for all $x, y, z \in \mathrm{T}$;

(4) a fuzzy lateral ideal of $T$ if $f([x y z]) \geqslant f(y)$ for all $x, y, z \in T$;

(5) a fuzzy ideal of $T$ if $f([x y z]) \geqslant \max \{f(x), f(y), f(z)\}$ for all $x, y, z \in T$. 
Note that a fuzzy subset $f$ is a fuzzy ideal of a ternary semigroup $T$ if and only if $f$ is a fuzzy left ideal, fuzzy right ideal and fuzzy lateral ideal of $\mathrm{T}$.

Definition 2.6. For any three fuzzy sets $f_{1}, f_{2}$, and $f_{3}$ of a ternary semigroup $T$, the product $\left[f_{1} \circ f_{2} \circ f_{3}\right]$ of $f_{1}, f_{2}$ and $f_{3}$ is defined by

$$
\left[f_{1} \circ f_{2} \circ f_{3}\right](y)=\sup _{y=\left[y_{1} y_{2} y_{3}\right]} \min \left\{f_{1}\left(y_{1}\right), f_{2}\left(y_{2}\right), f_{3}\left(y_{3}\right)\right\}
$$

Let $\mathcal{F}(T)$ be the set of all fuzzy subset of a ternary semigroup $T$. Note that $\mathcal{F}(T)$ is a ternary semigroup under the product defined in Definition 2.6.

\subsection{Pythagorean fuzzy sets}

Yager [21] and Yager and Abbasov [22] initiated the notion of Pythagorean fuzzy sets as follows.

Definition $2.7([21,22])$. Let $U$ be a universal set. A Pythagorean fuzzy set $\mathcal{P}:=\left\{\left\langle y, \mu_{\mathcal{P}}(y), v_{\mathcal{P}}(y)\right\rangle \mid y \in U\right\}$ where $\mu_{\mathcal{P}}: U \rightarrow[0,1]$ and $\nu_{\mathcal{P}}: U \rightarrow[0,1]$ represents the degree of membership and the degree of nonmembership of $y \in U$ to a set $\mathcal{P}$ with condition that $0 \leqslant\left(\mu_{\mathcal{P}}(y)\right)^{2}+\left(\nu_{\mathcal{P}}(y)\right)^{2} \leqslant 1$. For the sake of simplicity a Pythagorean fuzzy set $\mathcal{P}$ is denoted by $\mathcal{P}=\left(\mu_{\mathcal{P}}, \nu_{\mathcal{P}}\right)$.

Definition $2.8([21,22])$. Let $\mathcal{P}_{1}=\left(\mu_{\mathcal{P}_{1}}, v_{\mathcal{P}_{1}}\right)$ and $\mathcal{P}_{2}=\left(\mu_{\mathcal{P}_{2}}, \nu_{\mathcal{P}_{2}}\right)$ be Pythagorean fuzzy sets on a set $U$. Then for all $y \in U$,

(1) $\mathcal{P}_{1} \subseteq \mathcal{P}_{2} \Leftrightarrow \mu_{\mathcal{P}_{1}} \leqslant \mu_{\mathcal{P}_{2}}$ and $\nu_{\mathcal{P}_{2}} \leqslant \nu_{\mathcal{P}_{1}}$;

(2) $\mathcal{P}_{1}=\mathcal{P}_{2} \Leftrightarrow \mathcal{P}_{1} \subseteq \mathcal{P}_{2}$ and $\mathcal{P}_{2} \subseteq \mathcal{P}_{1}$;

(3) $\mathcal{P}_{1} \cup \mathcal{P}_{2}=\left(\mu_{\mathcal{P}_{1}} \cup \mu_{\mathcal{P}_{2}}, \nu_{\mathcal{P}_{1}} \cap v_{\mathcal{P}_{2}}\right)$;

(4) $\mathcal{P}_{1} \cap \mathcal{P}_{2}=\left(\mu_{\mathcal{P}_{1}} \cap \mu_{\mathcal{P}_{2}}, v_{\mathcal{P}_{1}} \cup \nu_{\mathcal{P}_{2}}\right)$.

Note that if $\mathcal{P}_{1}$ and $\mathcal{P}_{2}$ are Pythagorean fuzzy sets on a set $U$, then $\mathcal{P}_{1} \cup \mathcal{P}_{2}$ and $\mathcal{P}_{1} \cap \mathcal{P}_{2}$ are also Pythagorean fuzzy sets on $\mathrm{U}$.

\section{Main results}

3.1. Pythagorean fuzzy sets in ternary semigroups

Definition 3.1. A Pythagorean fuzzy set $\mathcal{P}=\left(\mu_{\mathcal{P}}, \nu_{\mathcal{P}}\right)$ is called

(1) a Pythagorean fuzzy ternary subsemigroup of $T$ if for all $y_{1}, y_{2}, y_{3} \in T$,

$$
\mu_{\mathcal{P}}\left(\left[y_{1} y_{2} y_{3}\right]\right) \geqslant \min \left\{\mu_{\mathcal{P}}\left(y_{1}\right), \mu_{\mathcal{P}}\left(y_{2}\right), \mu_{\mathcal{P}}\left(y_{3}\right)\right\} \quad \text { and } \quad v_{\mathcal{P}}\left(\left[y_{1} y_{2} y_{3}\right]\right) \leqslant \max \left\{v_{\mathcal{P}}\left(y_{1}\right), v_{\mathcal{P}}\left(y_{2}\right), v_{\mathcal{P}}\left(y_{3}\right)\right\}
$$

(2) a Pythagorean fuzzy left ideal of $T$ if for all $y_{1}, y_{2}, y_{3} \in T$,

$$
\mu_{\mathcal{P}}\left(\left[y_{1} y_{2} y_{3}\right]\right) \geqslant \mu_{\mathcal{P}}\left(y_{3}\right) \text { and } \nu_{\mathcal{P}}\left(\left[y_{1} y_{2} y_{3}\right]\right) \leqslant v_{\mathcal{P}}\left(y_{3}\right),
$$

(3) a Pythagorean fuzzy right ideal of $T$ if for all $y_{1}, y_{2}, y_{3} \in T$,

$$
\mu_{\mathcal{P}}\left(\left[y_{1} y_{2} y_{3}\right]\right) \geqslant \mu_{\mathcal{P}}\left(y_{1}\right) \text { and } \nu_{\mathcal{P}}\left(\left[y_{1} y_{2} y_{3}\right]\right) \leqslant v_{\mathcal{P}}\left(y_{1}\right),
$$

(4) a Pythagorean fuzzy lateral ideal of $\mathrm{T}$ if for all $\mathrm{y}_{1}, \mathrm{y}_{2}, \mathrm{y}_{3} \in \mathrm{T}$,

$$
\mu_{\mathcal{P}}\left(\left[y_{1} y_{2} y_{3}\right]\right) \geqslant \mu_{\mathcal{P}}\left(y_{2}\right) \text { and } \nu_{\mathcal{P}}\left(\left[y_{1} y_{2} y_{3}\right]\right) \leqslant \nu_{\mathcal{P}}\left(y_{2}\right),
$$

(5) a Pythagorean fuzzy ideal of $T$ if for all $y_{1}, y_{2}, y_{3} \in T$,

$$
\mu_{\mathcal{P}}\left(\left[y_{1} y_{2} y_{3}\right]\right) \geqslant \max \left\{\mu_{\mathcal{P}}\left(y_{1}\right), \mu_{\mathcal{P}}\left(y_{2}\right), \mu_{\mathcal{P}}\left(y_{3}\right)\right\} \quad \text { and } \quad v_{\mathcal{P}}\left(\left[y_{1} y_{2} y_{3}\right]\right) \leqslant \min \left\{v_{\mathcal{P}}\left(y_{1}\right), v_{\mathcal{P}}\left(y_{2}\right), v_{\mathcal{P}}\left(y_{3}\right)\right\}
$$


Theorem 3.2. Let $\mathrm{f}$ be a fuzzy subset of a ternary semigroup $\mathrm{T}$. Let $\mu_{\mathcal{P}}=\mathrm{f}$ and $\boldsymbol{\nu}_{\mathcal{P}}=1-\mathrm{f}$. Then $\mathcal{P}=\left(\mu_{\mathcal{P}}, \nu_{\mathcal{P}}\right)$ is a Pythagorean fuzzy set on T. Moreover, we have

(1) $f$ is a fuzzy ternary subsemigroup of $\mathrm{T}$ if and only if $\mathcal{P}$ is a Pythagorean fuzzy ternary subsemigroup of $\mathrm{T}$; and

(2) $\mathrm{f}$ is a fuzzy left ideal (respectively right ideal, lateral ideal, and ideal) of $\mathrm{T}$ if and only if $\mathcal{P}$ is a Pythagorean fuzzy left (respectively right ideal, lateral ideal, ideal) ideal of $\mathrm{T}$.

Proof. Let $f$ be a fuzzy subset of $\mathrm{T}$ and $\mathrm{y} \in \mathrm{T}$. Clearly,

$$
0 \leqslant(f(y))^{2}+((1-f)(y))^{2} \leqslant f(y)+(1-f)(y)=1 .
$$

Then $0 \leqslant\left(\mu_{\mathcal{P}}(y)\right)^{2}+\left(\nu_{\mathcal{P}}(y)\right)^{2} \leqslant 1$. Therefore $\mathcal{P}=\left(\mu_{\mathcal{P}}, \nu_{\mathcal{P}}\right)$ is a Pythagorean fuzzy set on $\mathrm{T}$.

(1) Let $f$ be a fuzzy ternary subsemigroup of $T$ and $y_{1}, y_{2}, y_{3} \in T$. Then

$$
\mu_{\mathcal{P}}\left(\left[y_{1} y_{2} y_{3}\right]\right)=f\left(\left[y_{1} y_{2} y_{3}\right]\right) \geqslant \min \left\{f\left(y_{1}\right), f\left(y_{2}\right), f\left(y_{3}\right)\right\}=\min \left\{\mu_{\mathcal{P}}\left(y_{1}\right), \mu_{\mathcal{P}}\left(y_{2}\right), \mu_{\mathcal{P}}\left(y_{3}\right)\right\}
$$

and

$$
\begin{aligned}
v_{\mathcal{P}}\left(\left[y_{1} y_{2} y_{3}\right]\right)=(1-f)\left(\left[y_{1} y_{2} y_{3}\right]\right) & =1-f\left(\left[y_{1} y_{2} y_{3}\right]\right) \\
& \leqslant 1-\min \left\{f\left(y_{1}\right), f\left(y_{2}\right), f\left(y_{3}\right)\right\} \\
& =\max \left\{(1-f)\left(y_{1}\right),(1-f)\left(y_{2}\right),(1-f)\left(y_{3}\right)\right\}=\max \left\{v_{\mathcal{P}}\left(y_{1}\right), v_{\mathcal{P}}\left(y_{2}\right), v_{\mathcal{P}}\left(y_{3}\right)\right\} .
\end{aligned}
$$

This implies that $\mathcal{P}$ is a Pythagorean fuzzy ternary subsemigroup of $T$. Conversely, assume that $\mathcal{P}$ is a Pythagorean fuzzy ternary subsemigroup of $T$. Since $f=\mu_{\mathcal{P}}$ and property of $\mu_{\mathcal{P}}$, this implies that $f$ is a fuzzy ternary subsemigroup of $\mathrm{T}$.

The proof of (2) is similar to the proof of (1).

Definition 3.3. Let $\mathcal{P}_{1}, \mathcal{P}_{2}$ and $\mathcal{P}_{3}$ be any three Pythagorean fuzzy sets on a ternary semigroup T. The product $\left[\mathcal{P}_{1} \circ \mathcal{P}_{2} \circ \mathcal{P}_{3}\right]$ of $\mathcal{P}_{1}, \mathcal{P}_{2}$, and $\mathcal{P}_{3}$ is defined by

$$
\left[\mathcal{P}_{1} \circ \mathcal{P}_{2} \circ \mathcal{P}_{3}\right]=\left(\left[\mu_{\mathcal{P}_{1}} \circ \mu_{\mathcal{P}_{2}} \circ \mu_{\mathcal{P}_{3}}\right],\left[\nu_{\mathcal{P}_{1}} \circ v_{\mathcal{P}_{2}} \circ v_{\mathcal{P}_{3}}\right]\right),
$$

where

$$
\left[\mu_{\mathcal{P}_{1}} \circ \mu_{\mathcal{P}_{2}} \circ \mu_{\mathcal{P}_{3}}\right](y)=\sup _{y=\left[y_{1} y_{2} y_{3}\right]} \min \left\{\mu_{\mathcal{P}_{1}}\left(y_{1}\right), \mu_{\mathcal{P}_{2}}\left(y_{2}\right), \mu_{\mathcal{P}_{3}}\left(y_{3}\right)\right\}
$$

and

$$
\left[v_{\mathcal{P}_{1}} \circ v_{\mathcal{P}_{2}} \circ v_{\mathcal{P}_{3}}\right](y)=\inf _{y=\left[y_{1} y_{2} y_{3}\right]} \max \left\{v_{\mathcal{P}_{1}}\left(y_{1}\right), v_{\mathcal{P}_{2}}\left(y_{2}\right), v_{\mathcal{P}_{3}}\left(y_{3}\right)\right\}
$$

Let $\mathcal{P F S}(\mathrm{T})$ be the set of all Pythagorean fuzzy sets on a ternary semigroup $\mathrm{T}$. Note that $\mathcal{P \mathcal { F }}(\mathrm{T})$ is a ternary semigroup under the product defined in Definition 3.3. Let $\mathcal{T}:=\left(\mu_{\mathcal{T}}, v_{\mathcal{T}}\right)$ be a Pythagorean fuzzy set on $T$ defined by $\mu_{\mathcal{T}}(y)=1$ and $\nu_{\mathcal{T}}(y)=0$ for all $y \in T$. The following theorem holds.

Theorem 3.4. Let $\mathcal{P}=\left(\mu_{\mathcal{P}}, v_{\mathcal{P}}\right)$ be a Pythagorean fuzzy set on a ternary semigroup $\mathrm{T}$.

(1) $\mathcal{P}$ is a Pythagorean fuzzy ternary subsemigroup of $\mathrm{T}$ if and only if $[\mathcal{P} \circ \mathcal{P} \circ \mathcal{P}] \subseteq \mathcal{P}$.

(2) $\mathcal{P}$ is a Pythagorean fuzzy left ideal of $\mathrm{T}$ if and only if $[\mathcal{T} \circ \mathcal{T} \circ \mathcal{P}] \subseteq \mathcal{P}$.

(3) $\mathcal{P}$ is a Pythagorean fuzzy right ideal of $\mathrm{T}$ if and only if $[\mathcal{P} \circ \mathcal{T} \circ \mathcal{T}] \subseteq \mathcal{P}$.

(4) $\mathcal{P}$ is a Pythagorean fuzzy lateral ideal of $\mathrm{T}$ if and only if $[\mathcal{T} \circ \mathcal{P} \circ \mathcal{T}] \subseteq \mathcal{P}$.

Proof.

(1) Assume that $\mathcal{P}$ is a Pythagorean fuzzy ternary subsemigroup of T. We have

$$
\left[\mu_{\mathcal{P}} \circ \mu_{\mathcal{P}} \circ \mu_{\mathcal{P}}\right](y)=\sup _{y=\left[y_{1} y_{2} y_{3}\right]} \min \left\{\mu_{\mathcal{P}}\left(y_{1}\right), \mu_{\mathcal{P}}\left(y_{2}\right), \mu_{\mathcal{P}}\left(y_{3}\right)\right\} \leqslant \mu_{\mathcal{P}}(y)
$$


and

$$
\left[v_{\mathcal{P}} \circ v_{\mathcal{P}} \circ v_{\mathcal{P}}\right](y)=\inf _{y=\left[y_{1} y_{2} y_{3}\right]} \max \left\{\mu_{\mathcal{P}}\left(y_{1}\right), \mu_{\mathcal{P}}\left(y_{2}\right), \mu_{\mathcal{P}}\left(y_{3}\right)\right\} \geqslant v_{\mathcal{P}}(y) .
$$

Hence $[\mathcal{P} \circ \mathcal{P} \circ \mathcal{P}] \subseteq \mathcal{P}$. Conversely, let $\mathrm{y}_{1}, \mathrm{y}_{2}, \mathrm{y}_{3} \in \mathrm{T}$.

$$
\begin{aligned}
\mu_{\mathcal{P}}\left(\left[y_{1} y_{2} y_{3}\right]\right) & \geqslant\left[\mu_{\mathcal{P}} \circ \mu_{\mathcal{P}} \circ \mu_{\mathcal{P}}\right]\left(\left[y_{1} y_{2} y_{3}\right]\right) \\
& =\sup _{[a b c]=\left[y_{1} y_{2} y_{3}\right]} \min \left\{\mu_{\mathcal{P}}(a), \mu_{\mathcal{P}}(b), \mu_{\mathcal{P}}(c)\right\} \geqslant \min \left\{\mu_{\mathcal{P}}\left(y_{1}\right), \mu_{\mathcal{P}}\left(y_{2}\right), \mu_{\mathcal{P}}\left(y_{3}\right)\right\}
\end{aligned}
$$

and

$$
\begin{aligned}
v_{\mathcal{P}}\left(\left[y_{1} y_{2} y_{3}\right]\right) & \leqslant\left[v_{\mathcal{P}} \circ v_{\mathcal{P}} \circ v_{\mathcal{P}}\right]\left(\left[y_{1} y_{2} y_{3}\right]\right) \\
& =\inf _{[a b c]=\left[y_{1} y_{2} y_{3}\right]} \max \left\{\mu_{\mathcal{P}}(a), \mu_{\mathcal{P}}(b), \mu_{\mathcal{P}}(c)\right\} \leqslant \max \left\{\mu_{\mathcal{P}}\left(y_{1}\right), \mu_{\mathcal{P}}\left(y_{2}\right), \mu_{\mathcal{P}}\left(y_{3}\right)\right\} .
\end{aligned}
$$

This implies that $\mathcal{P}$ is a Pythagorean fuzzy ternary subsemigroup of $\mathrm{T}$.

(2) Assume that $\mathcal{P}$ is a Pythagorean fuzzy left ideal of T. We have

$$
\left[\mu_{\mathcal{T}} \circ \mu_{\mathcal{T}} \circ \mu_{\mathcal{P}}\right](y)=\sup _{y=\left[y_{1} y_{2} y_{3}\right]} \min \left\{\mu_{\mathcal{T}}\left(y_{1}\right), \mu_{\mathcal{T}}\left(y_{2}\right), \mu_{\mathcal{P}}\left(y_{3}\right)\right\}=\sup _{y=\left[y_{1} y_{2} y_{3}\right]} \mu_{\mathcal{P}}\left(y_{3}\right) \leqslant \mu_{\mathcal{P}}(y)
$$

and

$$
\left[v_{\mathcal{T}} \circ v_{\mathcal{T}} \circ v_{\mathcal{P}}\right](y)=\inf _{y=\left[y_{1} y_{2} y_{3}\right]} \max \left\{v_{\mathcal{T}}\left(y_{1}\right), v_{\mathcal{T}}\left(y_{2}\right), v_{\mathcal{P}}\left(y_{3}\right)\right\}=\inf _{y=\left[y_{1} y_{2} y_{3}\right]} v_{\mathcal{P}}\left(y_{3}\right) \geqslant v_{\mathcal{P}}(y) .
$$

Hence $[\mathcal{T} \circ \mathcal{T} \circ \mathcal{P}] \subseteq \mathcal{P}$. Conversely, let $y_{1}, y_{2}, y_{3} \in T$.

$$
\begin{aligned}
\mu_{\mathcal{P}}\left(\left[y_{1} y_{2} y_{3}\right]\right) & \geqslant\left[\mu_{\mathcal{T}} \circ \mu_{\mathcal{T}} \circ \mu_{\mathcal{P}}\right]\left(\left[y_{1} y_{2} y_{3}\right]\right) \\
& =\sup _{[a b c]=\left[y_{1} y_{2} y_{3}\right]} \min \left\{\mu_{\mathcal{T}}(a), \mu_{\mathcal{T}}(b), \mu_{\mathcal{P}}(c)\right\}=\sup _{[a b c]=\left[y_{1} y_{2} y_{3}\right]} \mu_{\mathcal{P}}(c) \geqslant \mu_{\mathcal{P}}\left(y_{3}\right)
\end{aligned}
$$

and

$$
\begin{aligned}
v_{\mathcal{P}}\left(\left[y_{1} y_{2} y_{3}\right]\right) & \leqslant\left[v_{\mathcal{T}} \circ v_{\mathcal{T}} \circ v_{\mathcal{P}}\right]\left(\left[y_{1} y_{2} y_{3}\right]\right) \\
& =\inf _{[a b c]=\left[y_{1} y_{2} y_{3}\right]} \max \left\{v_{\mathcal{T}}(a), v_{\mathcal{T}}(b), v_{\mathcal{P}}(c)\right\}=\inf _{[a b c]=\left[y_{1} y_{2} y_{3}\right]} v_{\mathcal{P}}(c) \leqslant v_{\mathcal{P}}\left(y_{3}\right) .
\end{aligned}
$$

Then $\mu_{\mathcal{P}}\left(\left[y_{1} y_{2} y_{3}\right]\right) \geqslant \mu_{\mathcal{P}}\left(y_{3}\right)$ and $v_{\mathcal{P}}\left(\left[y_{1} y_{2} y_{3}\right]\right) \leqslant v_{\mathcal{P}}\left(y_{3}\right)$. This implies that $\mathcal{P}$ is a Pythagorean fuzzy left ideal of $\mathrm{T}$.

The proofs of (3) and (4) are similar to the proof of (2).

3.2. Rough Pythagorean fuzzy sets in ternary semigroups

Definition 3.5. An equivalence relation $\rho$ on a ternary semigroup $T$ is called a congruence if for all $x_{1}, x_{2}, x_{3}, y_{1}, y_{2}, y_{3} \in S$

$$
\left(x_{1}, y_{1}\right),\left(x_{2}, y_{2}\right),\left(x_{3}, y_{3}\right) \in \rho \Rightarrow\left(\left[x_{1} x_{2} x_{3}\right],\left[y_{1}, y_{2}, y_{3}\right]\right) \text {. }
$$

The congruence class of $x \in S$ is denoted by $[x]_{\rho}$. A congruence $\rho$ of $T$ is called complete if $\left[y_{1}\right]_{\rho}\left[y_{2}\right]_{\rho}\left[y_{3}\right]_{\rho}=$ $\left[y_{1} y_{2} y_{3}\right]_{\rho}$ for all $y_{1}, y_{2}, y_{3} \in T$.

Definition 3.6. Let $\rho$ be a congruence and $\mathcal{P}=\left\{\left\langle y, \mu_{\mathcal{P}}(y), \nu_{\mathcal{P}}(y)\right\rangle \mid y \in T\right\}$ be the Pythagorean fuzzy set on a ternary semigroup $T$.

(1) The lower approximation is defined as

$$
\underline{\operatorname{App}}(\mathcal{P})=\left\{<y, \underline{\mu_{\mathcal{P}}}(y), \underline{\nu_{\mathcal{P}}}(y)>\mid y \in T\right\},
$$

where $\underline{\mu_{\mathcal{P}}}(y)=\inf _{y^{\prime} \in[y]_{\rho}} \mu_{\mathcal{P}}\left(y^{\prime}\right)$ and $\underline{v_{\mathcal{P}}}(y)=\sup _{y^{\prime} \in[y]_{\rho}} v_{\mathcal{P}}\left(y^{\prime}\right)$ with the condition that

$$
0 \leqslant\left(\underline{\mu_{\mathcal{P}}}(y)\right)^{2}+\left(\underline{\nu_{\mathcal{P}}}(y)\right)^{2} \leqslant 1 .
$$


(2) The upper approximation is defined as

$$
\overline{\operatorname{App}}(\mathcal{P})=\left\{<y, \overline{\mu_{\mathcal{P}}}(y), \overline{v_{\mathcal{P}}}(y)>\mid y \in T\right\},
$$

where $\overline{\mu_{\mathcal{P}}}(y)=\sup _{y^{\prime} \in[y]_{\rho}} \mu_{\mathcal{P}}\left(y^{\prime}\right)$ and $\overline{v_{\mathcal{P}}}(y)=\inf _{y^{\prime} \in[y]_{\rho}} v_{\mathcal{P}}\left(y^{\prime}\right)$ with the condition that

$$
0 \leqslant\left(\overline{\mu_{\mathcal{P}}}(\mathrm{y})\right)^{2}+\left(\overline{\nu_{\mathcal{P}}}(\mathrm{y})\right)^{2} \leqslant 1
$$

(3) Rough Pythagorean fuzzy set of T is defined by

$$
\operatorname{App}(\mathcal{P})=(\underline{\operatorname{App}}(\mathcal{P}), \overline{\operatorname{App}}(\mathcal{P})) .
$$

Example 3.7. Define a relation $\rho$ on a ternary semigroup $\mathbb{Z}^{-}$under the usual multiplication by

$$
x \rho y \Leftrightarrow 2 \mid x-y \text { for all } x, y \in \mathbb{Z}^{-} .
$$

It is easy to show that $\rho$ is a congruence on $\mathbb{Z}^{-}$. Let $\mu_{\mathcal{P}}(y)=-\frac{1}{y}$ and $\nu_{\mathcal{P}}(y)=1+\frac{1}{y}$ for all $y \in \mathbb{Z}^{-}$. Then

$$
0 \leqslant\left(\mu_{\mathcal{P}}(y)\right)^{2}+\left(\nu_{\mathcal{P}}(y)\right)^{2}=\left(-\frac{1}{y}\right)^{2}+\left(1+\frac{1}{y}\right)^{2}=1+2\left(\frac{1}{y}+\frac{1}{y^{2}}\right) \leqslant 1
$$

for all $y \in \mathbb{Z}^{-}$, this implies that $\mathcal{P}$ is a Pythagorean fuzzy set of $T$. We have

$$
\underline{\operatorname{App}}(\mathcal{P})=\left\{\left\langle y, \underline{\mu_{\mathcal{P}}}(y), \underline{v_{\mathcal{P}}}(y)>\right| y \in T\right\}=\{<y, 0,1>\mid y \in T\}
$$

and

$$
\overline{\operatorname{App}}(\mathcal{P})=\left\{<y, \overline{\mu_{\mathcal{P}}}(y), \overline{v_{\mathcal{P}}}(y)>\mid y \in T\right\}=\{<y, 1,0>\mid y \text { is odd }\} \cup\left\{<y, \frac{1}{2}, \frac{1}{2}>\mid y \text { is even }\right\}
$$

Theorem 3.8. Let $\rho$ be a congruence relation on a ternary semigroup $\mathrm{T}$ and $\mathcal{P}$ be a Pythagorean fuzzy set of $\mathrm{T}$.

(1) $\overline{\operatorname{App}}(\mathcal{P})$ is a Pythagorean fuzzy set of $\mathrm{T}$.

(2) $\operatorname{App}(\mathcal{P})$ is a Pythagorean fuzzy set of $\mathrm{T}$.

Proof.

(1) Let $y \in T$. Then

$$
\begin{aligned}
\left(\overline{\mu_{\mathcal{P}}}(y)\right)^{2}+\left(\overline{\mathcal{V}_{\mathcal{P}}}(y)\right)^{2} & =\left(\sup _{y^{\prime} \in[y]_{\rho}} \mu_{\mathcal{P}}\left(y^{\prime}\right)\right)^{2}+\left(\inf _{y^{\prime} \in[y]_{\rho}} \nu_{\mathcal{P}}\left(y^{\prime}\right)\right)^{2} \\
& =\sup _{y^{\prime} \in[y]_{\rho}}\left(\mu_{\mathcal{P}}\left(y^{\prime}\right)\right)^{2}+\inf _{y^{\prime} \in[y]_{\rho}}\left(\nu_{\mathcal{P}}\left(y^{\prime}\right)\right)^{2} \\
& \leqslant \sup _{y^{\prime} \in[y]_{\rho}}\left(\mu_{\mathcal{P}}\left(y^{\prime}\right)\right)^{2}+\inf _{y^{\prime} \in[y]_{\rho}}\left(1-\left(\mu_{\mathcal{P}}\left(y^{\prime}\right)\right)^{2}\right) \\
& \leqslant \sup _{y^{\prime} \in[y]_{\rho}}\left(\mu_{\mathcal{P}}\left(y^{\prime}\right)\right)^{2}+1-\sup _{y^{\prime} \in[y]_{\rho}}\left(\mu_{\mathcal{P}}\left(y^{\prime}\right)\right)^{2}=1 .
\end{aligned}
$$

This implies that $0 \leqslant\left(\overline{\mu_{\mathcal{P}}}(y)\right)^{2}+\left(\overline{v_{\mathcal{P}}}(y)\right)^{2} \leqslant 1$. Therefore, $\overline{\operatorname{App}}(\mathcal{P})$ is a Pythagorean fuzzy set of $\mathrm{T}$.

The proof of (2) is similar to the proof of (1).

Theorem 3.9. Let $\rho$ be a congruence on a ternary semigroup $T$ and $\mathcal{P}_{1}=\left(\mu_{\mathcal{P}_{1}}, \nu_{\mathcal{P}_{1}}\right)$ and $\mathcal{P}_{2}=\left(\mu_{\mathcal{P}_{2}}, \nu_{\mathcal{P}_{2}}\right)$ be Pythagorean fuzzy sets on $\mathrm{T}$. The following statements hold.

(1) If $\mathcal{P}_{1} \subseteq \mathcal{P}_{2}$, then $\overline{\operatorname{App}}\left(\mathcal{P}_{1}\right) \subseteq \overline{\operatorname{App}}\left(\mathcal{P}_{2}\right)$ and $\underline{\operatorname{App}}\left(\mathcal{P}_{1}\right) \subseteq \underline{\operatorname{App}}\left(\mathcal{P}_{2}\right)$.

(2) $\overline{\operatorname{App}}\left(\mathcal{P}_{1} \cap \mathcal{P}_{2}\right) \subseteq \overline{\operatorname{App}}\left(\mathcal{P}_{1}\right) \cap \overline{\operatorname{App}}\left(\mathcal{P}_{2}\right)$. 
(3) $\overline{\operatorname{App}}\left(\mathcal{P}_{1} \cup \mathcal{P}_{2}\right)=\overline{\operatorname{App}}\left(\mathcal{P}_{1}\right) \cup \overline{\operatorname{App}}\left(\mathcal{P}_{2}\right)$.

(4) $\operatorname{App}\left(\mathcal{P}_{1} \cap \mathcal{P}_{2}\right)=\operatorname{App}\left(\mathcal{P}_{1}\right) \cap \operatorname{App}\left(\mathcal{P}_{2}\right)$.

(5) $\overline{\operatorname{App}}\left(\mathcal{P}_{1}\right) \cup \underline{A p p}\left(\overline{\left.\mathcal{P}_{2}\right) \subseteq \underline{A p p}} \overline{\left(\mathcal{P}_{1} \cup\right.} \mathcal{P}_{2}\right)$.

Proof.

(1) Assume that $\mathcal{P}_{1} \subseteq \mathcal{P}_{2}$. Then $\mu_{\mathcal{P}_{1}} \leqslant \mu_{\mathcal{P}_{2}}$ and $\nu_{\mathcal{P}_{2}} \leqslant \nu_{\mathcal{P}_{1}}$. Thus for all $y \in T$, we have

$$
\overline{\mu_{\mathcal{P}_{1}}}(y)=\sup _{y^{\prime} \in[y]_{\rho}} \mu_{\mathcal{P}_{1}}\left(y^{\prime}\right) \leqslant \sup _{y^{\prime} \in[y]_{\rho}} \mu_{\mathcal{P}_{2}}\left(y^{\prime}\right)=\overline{\mu_{\mathcal{P}_{2}}}(y)
$$

and

$$
\overline{v_{\mathcal{P}_{1}}}(y)=\inf _{y^{\prime} \in[y]_{\rho}} v_{\mathcal{P}_{1}}\left(y^{\prime}\right) \geqslant \inf _{y^{\prime} \in[y]_{\rho}} v_{\mathcal{P}_{2}}\left(y^{\prime}\right)=\overline{v_{\mathcal{P}_{2}}}(y) .
$$

This implies that $\overline{\operatorname{App}}\left(\mathcal{P}_{1}\right) \subseteq \overline{\operatorname{App}}\left(\mathcal{P}_{2}\right)$. Similarly, we have $\underline{\operatorname{App}}\left(\mathcal{P}_{1}\right) \subseteq \underline{\operatorname{App}}\left(\mathcal{P}_{2}\right)$.

(2) Since $\mathcal{P}_{1} \cap \mathcal{P}_{2} \subseteq \mathcal{P}_{1}$ and $\mathcal{P}_{1} \cap \mathcal{P}_{2} \subseteq \mathcal{P}_{2}, \overline{\operatorname{App}}\left(\mathcal{P}_{1} \cap \mathcal{P}_{2}\right) \subseteq \overline{\operatorname{App}}\left(\mathcal{P}_{1}\right) \cap \overline{\overline{A p p}}\left(\mathcal{P}_{2}\right)$ by (1).

(3) Note that

$$
\overline{\operatorname{App}}\left(\mathcal{P}_{1}\right) \cup \overline{\operatorname{App}}\left(\mathcal{P}_{2}\right)=\left(\overline{\mu_{\mathcal{P}_{1}}} \cup \overline{\mu_{\mathcal{P}_{2}}}, \overline{\overline{\mathcal{P}}_{1}} \cap \overline{\mathcal{V}_{2}}\right)
$$

and

$$
\overline{\operatorname{App}}\left(\mathcal{P}_{1} \cup \mathcal{P}_{2}\right)=\left(\overline{\mu_{\mathcal{P}_{1} \cup \mathcal{P}_{2}}}, \overline{\mathcal{V}_{\mathcal{P}_{1} \cup \mathcal{P}_{2}}}\right) .
$$

Let $y \in T$. Then

$$
\begin{aligned}
\left(\overline{\mu_{\mathcal{P}_{1}}} \cup \overline{\mu_{\mathcal{P}_{2}}}\right)(y) & =\max \left\{\overline{\mu_{\mathcal{P}_{1}}}(y), \overline{\mu_{\mathcal{P}_{2}}}(y)\right\} \\
& =\max \left\{\sup _{y^{\prime} \in[y]_{\rho}} \mu_{\mathcal{P}_{1}}\left(y^{\prime}\right), \sup _{y^{\prime} \in[y]_{\rho}} \mu_{\mathcal{P}_{2}}\left(y^{\prime}\right)\right\} \\
& =\sup _{y^{\prime} \in[y]_{\rho}} \max \left\{\mu_{\mathcal{P}_{1}}\left(y^{\prime}\right), \mu_{\mathcal{P}_{2}}\left(y^{\prime}\right)\right\}=\sup _{y^{\prime} \in[y]_{\rho}} \mu_{\mathcal{P}_{1} \cup \mathcal{P}_{2}}\left(y^{\prime}\right)=\overline{\mu_{\mathcal{P}_{1} \cup \mathcal{P}_{2}}}(y)
\end{aligned}
$$

and

$$
\begin{aligned}
\left(\overline{\mathcal{V}_{1}} \cap \overline{\mathcal{V}_{\mathcal{P}_{2}}}\right)(y) & =\min \left\{\overline{\mathcal{v}_{1}}(y), \overline{\mathcal{V}_{2}}(y)\right\} \\
& =\min \left\{\inf _{y^{\prime} \in[y]_{\rho}} \mu_{\mathcal{P}_{1}}\left(y^{\prime}\right), \inf _{y^{\prime} \in[y]_{\rho}} \mu_{\mathcal{P}_{2}}\left(y^{\prime}\right)\right\} \\
& =\inf _{y^{\prime} \in[y]_{\rho}} \min \left\{v_{\mathcal{P}_{1}}\left(y^{\prime}\right), \nu_{\mathcal{P}_{2}}\left(y^{\prime}\right)\right\}=\inf _{y^{\prime} \in[y]_{\rho}} v_{\mathcal{P}_{1} \cup \mathcal{P}_{2}}\left(y^{\prime}\right)=\overline{v_{\mathcal{P}_{1} \cup \mathcal{P}_{2}}}(y) .
\end{aligned}
$$

(4) Note that

and

$$
\underline{\operatorname{App}}\left(\mathcal{P}_{1}\right) \cap \underline{\operatorname{App}}\left(\mathcal{P}_{2}\right)=\left(\underline{\mu_{\mathcal{P}_{1}}} \cap \underline{\mu_{\mathcal{P}_{2}}}, \underline{v_{\mathcal{P}_{1}}} \cup \underline{v_{\mathcal{P}_{2}}}\right)
$$

Let $y \in T$. Then

$$
\underline{\operatorname{App}}\left(\mathcal{P}_{1} \cap \mathcal{P}_{2}\right)=\left(\underline{\mu_{\mathcal{P}_{1} \cap \mathcal{P}_{2}}}, \underline{\nu_{\mathcal{P}_{1} \cap \mathcal{P}_{2}}}\right) .
$$

$$
\begin{aligned}
\left.\underline{\left(\mu_{\mathcal{P}_{1}}\right.} \cap \underline{\mu_{\mathcal{P}_{2}}}\right)(y) & =\min \left\{\underline{\mu_{\mathcal{P}_{1}}}(y), \underline{\mu_{\mathcal{P}_{2}}}(y)\right\} \\
& =\min \left\{\underline{\inf _{y^{\prime} \in[y]_{\rho}}} \underline{\left.\mu_{\mathcal{P}_{1}}\left(y^{\prime}\right), \inf _{y^{\prime} \in[y]_{\rho}} \mu_{\mathcal{P}_{2}}\left(y^{\prime}\right)\right\}}\right. \\
& =\inf _{y^{\prime} \in[y]_{\rho}} \min \left\{\mu_{\mathcal{P}_{1}}\left(y^{\prime}\right), \mu_{\mathcal{P}_{2}}\left(y^{\prime}\right)\right\}=\inf _{y^{\prime} \in[y]_{\rho}} \mu_{\mathcal{P}_{1} \cap \mathcal{P}_{2}}\left(y^{\prime}\right)=\underline{\mu_{\mathcal{P}_{1} \cap \mathcal{P}_{2}}}(y)
\end{aligned}
$$

and

$$
\begin{aligned}
\left(\underline{v_{\mathcal{P}_{1}}} \cup \underline{v_{\mathcal{P}_{2}}}\right)(y) & =\max \left\{\underline{v_{\mathcal{P}_{1}}}(y), \underline{v_{\mathcal{P}_{2}}}(y)\right\} \\
& =\max \left\{\sup _{y^{\prime} \in[y]_{\rho}} \mu_{\mathcal{P}_{1}}\left(y^{\prime}\right), \sup _{y^{\prime} \in[y]_{\rho}} \mu_{\mathcal{P}_{2}}\left(y^{\prime}\right)\right\} \\
& =\sup _{y^{\prime} \in[y]_{\rho}} \max \left\{v_{\mathcal{P}_{1}}\left(y^{\prime}\right), v_{\mathcal{P}_{2}}\left(y^{\prime}\right)\right\}=\sup _{y^{\prime} \in[y]_{\rho}} v_{\mathcal{P}_{1} \cap \mathcal{P}_{2}}\left(y^{\prime}\right)=\underline{v_{\mathcal{P}_{1} \cap \mathcal{P}_{2}}}(y) .
\end{aligned}
$$


(5) Since $\mathcal{P}_{1} \subseteq \mathcal{P}_{1} \cup \mathcal{P}_{2}$ and $\mathcal{P}_{1} \subseteq \mathcal{P}_{1} \cup \mathcal{P}_{2}, \underline{\operatorname{App}}\left(\mathcal{P}_{1}\right) \cup \underline{\operatorname{App}}\left(\mathcal{P}_{2}\right) \subseteq \underline{\operatorname{App}}\left(\mathcal{P}_{1} \cup \mathcal{P}_{2}\right)$ by (1).

Example 3.10. Let $\rho$ be a congruence on a ternary semigroup $\mathbb{Z}^{-}$under the usual multiplication defined by

$$
x \rho y \Leftrightarrow 2 \mid x-y \text { for all } x, y \in \mathbb{Z}^{-} .
$$

(1) Let $\mathcal{P}_{1}=\left(\mu_{\mathcal{P}_{1}}, \nu_{\mathcal{P}_{1}}\right)$ and $\mathcal{P}_{2}=\left(\mu_{\mathcal{P}_{2}}, \nu_{\mathcal{P}_{2}}\right)$ be Pythagorean fuzzy sets on $T$ defined by

$$
\begin{aligned}
\mu_{\mathcal{P}_{1}}(-1) & =\mu_{\mathcal{P}_{1}}(-4)=1, \mu_{\mathcal{P}_{1}}(-2)=\mu_{\mathcal{P}_{1}}(-3)=0, \\
\mu_{\mathcal{P}_{2}}(-1) & =\mu_{\mathcal{P}_{2}}(-4)=0, \mu_{\mathcal{P}_{2}}(-2)=\mu_{\mathcal{P}_{2}}(-3)=1, \\
\mu_{\mathcal{P}_{1}}(x) & =\mu_{\mathcal{P}_{2}}(y)=0 \text { for all } y \in \mathbb{Z}^{-} \backslash\{-1,-2,-3,-4\},
\end{aligned}
$$

and

$$
v_{\mathcal{P}_{1}}(x)=v_{\mathcal{P}_{2}}(y)=0 \text { for all } y \in \mathbb{Z}^{-}
$$

We have

$$
\overline{\operatorname{App}}\left(\mathcal{P}_{1} \cap \mathcal{P}_{2}\right)=\{<y, 0,0>\mid y \in T\}
$$

and

$$
\overline{\operatorname{App}}\left(\mathcal{P}_{1}\right) \cap \overline{\operatorname{App}}\left(\mathcal{P}_{2}\right)=\{<y, 1,0>\mid y \in T\} .
$$

This implies that the converse of (2) in Theorem 3.9 is not true in general.

(2) Let $\mathcal{P}_{1}=\left(\mu_{\mathcal{P}_{1}}, v_{\mathcal{P}_{1}}\right)$ and $\mathcal{P}_{2}=\left(\mu_{\mathcal{P}_{2}}, \nu_{\mathcal{P}_{2}}\right)$ be Pythagorean fuzzy sets on $T$ defined by for all $x \in \mathbb{Z}^{-}$,

$$
\mu_{\mathcal{P}_{1}}(y)=\left\{\begin{array}{ll}
0, & \text { if } y=-1 \text { or } y=-2, \\
1, & \text { otherwise, }
\end{array} \text { and } \mu_{\mathcal{P}_{2}}(x)= \begin{cases}1, & \text { if } y=-1 \text { or } y=-2, \\
0, & \text { otherwise }\end{cases}\right.
$$

and

$$
v_{\mathcal{P}_{1}}(y)=v_{\mathcal{P}_{2}}(y)=0 \text { for all } y \in \mathbb{Z}^{-} .
$$

We have

$$
\underline{\operatorname{App}}\left(\mathcal{P}_{1}\right) \cup \underline{\operatorname{App}}\left(\mathcal{P}_{2}\right)=\{<y, 0,0>\mid y \in T\}
$$

and

$$
\left.\underline{\operatorname{App}}\left(\mathcal{P}_{1} \cup \mathcal{P}_{2}\right)=\{<y, 1,0\rangle \mid y \in T\right\} .
$$

This implies that the converse of (5) in Theorem 3.9 is not true in general.

Theorem 3.11. Let $\rho$ be a complete congruence on a ternary semigroup $T$ and $\mathcal{P}_{1}=\left(\mu_{\mathcal{P}_{1}}, \nu_{\mathcal{P}_{1}}\right), \mathcal{P}_{2}=\left(\mu_{\mathcal{P}_{2}}, \nu_{\mathcal{P}_{2}}\right)$ and $\mathcal{P}_{3}=\left(\mu_{\mathcal{P}_{3}}, \nu_{\mathcal{P}_{3}}\right)$ be Pythagorean fuzzy sets on $\mathrm{T}$. Then

$$
\overline{\operatorname{App}}\left(\left[\mathcal{P}_{1} \circ \mathcal{P}_{2} \circ \mathcal{P}_{3}\right]\right) \subseteq\left[\overline{\operatorname{App}}\left(\mathcal{P}_{1}\right) \circ \overline{\operatorname{App}}\left(\mathcal{P}_{2}\right) \circ \overline{\operatorname{App}}\left(\mathcal{P}_{3}\right)\right]
$$

Proof. Obviously,

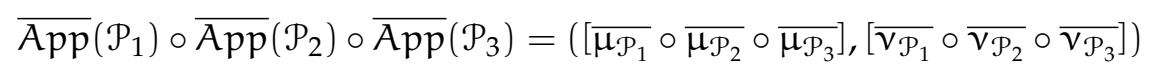

and

$$
\overline{\operatorname{App}}\left(\left[\mathcal{P}_{1} \circ \mathcal{P}_{2} \circ \mathcal{P}_{3}\right]\right)=\left(\left[\overline{\mu_{\mathcal{P}_{1}} \circ \mu_{\mathcal{P}_{2}} \circ \mu_{\mathcal{P}_{3}}}\right],\left[\overline{\nu_{\mathcal{P}_{1}} \circ \mathcal{V}_{\mathcal{P}_{2}} \circ \mathcal{V}_{\mathcal{P}_{3}}}\right]\right) .
$$

Let $y \in S$. Since $\left[y_{1}\right]_{\rho}\left[y_{2}\right]_{\rho}\left[y_{3}\right]_{\rho}=\left[y_{1} y_{2} y_{3}\right]_{\rho}$, we have

$$
\begin{aligned}
{\left[\overline{\mu_{\mathcal{P}_{1}}} \circ \overline{\mu_{\mathcal{P}_{2}}} \circ \overline{\mu_{\mathcal{P}_{3}}}\right](y) } & =\sup _{y=\left[y_{1} y_{2} y_{3}\right]} \min \left\{\overline{\mu_{\mathcal{P}_{1}}}\left(y_{1}\right), \overline{\mu_{\mathcal{P}_{2}}}\left(y_{2}\right), \overline{\mu_{\mathcal{P}_{3}}}\left(y_{3}\right)\right\} \\
& =\sup _{y=\left[y_{1} y_{2} y_{3}\right]} \min \left\{\operatorname { s u p } _ { y _ { 1 } ^ { \prime } \in [ y _ { 1 } ] _ { \rho } } \left(\mu_{\mathcal{P}}\left(y_{1}^{\prime}\right), \sup _{y_{2}^{\prime} \in\left[y_{2}\right]_{\rho}}\left(\mu_{\mathcal{P}}\left(y_{2}^{\prime}\right), \sup _{y_{3}^{\prime} \in\left[y_{3}\right]_{\rho}}\left(\mu_{\mathcal{P}}\left(y_{3}^{\prime}\right)\right\}\right.\right.\right.
\end{aligned}
$$




$$
\begin{aligned}
& \geqslant \sup _{y=\left[y_{1} y_{2} y_{3}\right]} \sup _{\left[y_{1}^{\prime} y_{2}^{\prime} y_{3}^{\prime}\right] \in\left[y_{1} y_{2} y_{3}\right]_{\rho}} \min \left\{\mu_{\mathcal{P}}\left(y_{1}^{\prime}\right), \mu_{\mathcal{P}}\left(y_{2}^{\prime}\right), \mu_{\mathcal{P}}\left(y_{3}^{\prime}\right)\right\} \\
& =\sup _{\left[y_{1}^{\prime} y_{2}^{\prime} y_{3}^{\prime}\right] \in[y]_{\rho}} \min \left\{\mu_{\mathcal{P}}\left(y_{1}^{\prime}\right), \mu_{\mathcal{P}}\left(y_{2}^{\prime}\right), \mu_{\mathcal{P}}\left(y_{3}^{\prime}\right)\right\} \\
& =\sup _{x \in[y]_{\rho}, x=\left[y_{1}^{\prime} y_{2}^{\prime} y_{3}^{\prime}\right]} \min \left\{\mu_{\mathcal{P}}\left(y_{1}^{\prime}\right), \mu_{\mathcal{P}}\left(y_{2}^{\prime}\right), \mu_{\mathcal{P}}\left(y_{3}^{\prime}\right)\right\} \\
& =\sup _{x \in[y]_{\rho}}\left\{\sup _{x=\left[y_{1}^{\prime} y_{2}^{\prime} y_{3}^{\prime}\right]} \min \left\{\mu_{\mathcal{P}}\left(y_{1}^{\prime}\right), \mu_{\mathcal{P}}\left(y_{2}^{\prime}\right), \mu_{\mathcal{P}}\left(y_{3}^{\prime}\right)\right\}\right\} \\
& =\sup _{x \in[y]_{\rho}}\left[\mu_{\mathcal{P}_{1}} \circ \mu_{\mathcal{P}_{2}} \circ \mu_{\mathcal{P}_{3}}\right](x)=\left[\overline{\left.\mu_{\mathcal{P}_{1}} \circ \mu_{\mathcal{P}_{2}} \circ \mu_{\mathcal{P}_{3}}\right]}(y) .\right.
\end{aligned}
$$

This implies that $\left[\overline{\left.\mu_{\mathcal{P}_{1}} \circ \mu_{\mathcal{P}_{2}} \circ \mu_{\mathcal{P}_{3}}\right]}(y) \leqslant\left[\overline{\mu_{\mathcal{P}_{1}}} \circ \overline{\mu_{\mathcal{P}_{2}}} \circ \overline{\mu_{\mathcal{P}_{3}}}\right](y)\right.$.

$$
\begin{aligned}
& {\left[\overline{\mathcal{V}_{\mathcal{P}_{1}}} \circ \overline{\mathcal{V}_{\mathcal{P}_{2}}} \circ \overline{\mathcal{V}_{\mathcal{P}_{3}}}\right](y)=\inf _{y=\left[y_{1} y_{2} y_{3}\right]} \max \left\{\overline{\mathcal{V}_{1}}\left(y_{1}\right), \overline{\mathcal{V}_{\mathcal{P}_{2}}}\left(y_{2}\right), \overline{v_{\mathcal{P}_{3}}}\left(y_{3}\right)\right\}} \\
& =\inf _{y=\left[y_{1} y_{2} y_{3}\right]} \max \left\{\operatorname { i n f } _ { y _ { 1 } ^ { \prime } \in [ y _ { 1 } ] _ { \rho } } \left(v_{\mathcal{P}}\left(y_{1}^{\prime}\right), \inf _{y_{2}^{\prime} \in\left[y_{2}\right]_{\rho}}\left(v_{\mathcal{P}}\left(y_{2}^{\prime}\right), \inf _{y_{3}^{\prime} \in\left[y_{3}\right]_{\rho}}\left(v_{\mathcal{P}}\left(y_{3}^{\prime}\right)\right\}\right.\right.\right. \\
& \leqslant \inf _{y=\left[y_{1} y_{2} y_{3}\right]} \inf _{\left[y_{1}^{\prime} y_{2}^{\prime} y_{3}^{\prime}\right] \in\left[y_{1} y_{2} y_{3}\right]_{\rho}} \max \left\{v_{\mathcal{P}}\left(y_{1}^{\prime}\right), v_{\mathcal{P}}\left(y_{2}^{\prime}\right), v_{\mathcal{P}}\left(y_{3}^{\prime}\right)\right\} \\
& =\inf _{\left[y_{1}^{\prime} y_{2}^{\prime} y_{3}^{\prime}\right] \in[y]_{\rho}} \max \left\{v_{\mathcal{P}}\left(y_{1}^{\prime}\right), v_{\mathcal{P}}\left(y_{2}^{\prime}\right), v_{\mathcal{P}}\left(y_{3}^{\prime}\right)\right\} \\
& =\inf _{x \in[y]_{\rho}, x=\left[y_{1}^{\prime} y_{2}^{\prime} y_{3}^{\prime}\right]} \max \left\{v_{\mathcal{P}}\left(y_{1}^{\prime}\right), v_{\mathcal{P}}\left(y_{2}^{\prime}\right), v_{\mathcal{P}}\left(y_{3}^{\prime}\right)\right\} \\
& =\inf _{x \in[y]_{\rho}}\left\{\inf _{x=\left[y_{1}^{\prime} y_{2}^{\prime} y_{3}^{\prime}\right]} \max \left\{v_{\mathcal{P}}\left(y_{1}^{\prime}\right), v_{\mathcal{P}}\left(y_{2}^{\prime}\right), v_{\mathcal{P}}\left(y_{3}^{\prime}\right)\right\}\right\} \\
& =\inf _{x \in[y]_{\rho}}\left[v_{\mathcal{P}_{1}} \circ v_{\mathcal{P}_{2}} \circ v_{\mathcal{P}_{3}}\right](x)=\left[\overline{\left.v_{\mathcal{P}_{1}} \circ v_{\mathcal{P}_{2}} \circ v_{\mathcal{P}_{3}}\right]}(y)\right. \text {. }
\end{aligned}
$$

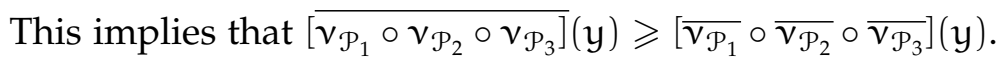

Therefore, $\overline{\operatorname{App}}\left(\left[\mathcal{P}_{1} \circ \mathcal{P}_{2} \circ \mathcal{P}_{3}\right]\right) \subseteq\left[\overline{\operatorname{App}}\left(\mathcal{P}_{1}\right) \circ \overline{\operatorname{App}}\left(\mathcal{P}_{2}\right) \circ \overline{\operatorname{App}}\left(\mathcal{P}_{3}\right)\right]$.

3.3. Rough Pythagorean fuzzy ideals in ternary semigroups

Theorem 3.12. Let $\rho$ be a congruence relation on a ternary semigroup $\mathrm{T}$ and $\mathcal{P}$ be a Pythagorean fuzzy set of $\mathrm{T}$.

(1) If $\mathcal{P}$ is a Pythagorean fuzzy left ideal (respectively Pythagorean fuzzy right ideal and Pythagorean fuzzy lateral idea) of $\mathrm{T}$, then $\overline{\operatorname{App}}(\mathcal{P})$ is a Pythagorean fuzzy left ideal (respectively Pythagorean fuzzy right ideal and Pythagorean fuzzy lateral ideal) of $\mathrm{T}$.

(2) If $\rho$ is complete and $\mathcal{P}$ is a Pythagorean fuzzy left ideal (respectively Pythagorean fuzzy right ideal and Pythagorean fuzzy lateral ideal) of $\mathrm{T}$, then $\mathrm{App}(\mathcal{P})$ is a Pythagorean fuzzy left ideal (respectively Pythagorean fuzzy right ideal and Pythagorean fuzzy lateral ideal) of $\mathrm{T}$.

Proof.

(1) Let $y_{1}, y_{2}, y_{3} \in T$.

$$
\begin{aligned}
\overline{\mu_{\mathcal{P}}}\left(\left[y_{1} y_{2} y_{3}\right]\right)=\sup _{y \in\left[y_{1} y_{2} y_{3}\right]_{\rho}} \mu_{\mathcal{P}}(y) & \geqslant \sup _{y \in\left[y_{1}\right]_{\rho}\left[y_{2}\right]_{\rho}\left[y_{3}\right]_{\rho}} \mu_{\mathcal{P}}(y) \\
& =\sup _{[a b c] \in\left[y_{1}\right]_{\rho}\left[y_{2}\right]_{\rho}\left[y_{3}\right]_{\rho}} \mu_{\mathcal{P}}([a b c]) \\
& \geqslant \sup _{[a b c] \in\left[y_{1}\right]_{\rho}\left[y_{2}\right]_{\rho}\left[y_{3}\right]_{\rho}} \mu_{\mathcal{P}}(c)=\sup _{c \in\left[y_{3}\right]_{\rho}} \mu_{\mathcal{P}}(c)=\overline{\mu_{\mathcal{P}}}\left(y_{3}\right)
\end{aligned}
$$


and

$$
\begin{aligned}
\overline{v_{\mathcal{P}}}\left(\left[y_{1} y_{2} y_{3}\right]\right)=\inf _{y \in\left[y_{1} y_{2} y_{3}\right]_{\rho}} v_{\mathcal{P}}(y) & \leqslant \inf _{y \in\left[y_{1}\right]_{\rho}\left[y_{2}\right]_{\rho}\left[y_{3}\right]_{\rho}} v_{\mathcal{P}}(y) \\
& =\inf _{[a b c] \in\left[y_{1}\right]_{\rho}\left[y_{2}\right]_{\rho}\left[y_{3}\right]_{\rho}} v_{\mathcal{P}}([a b c]) \\
& \leqslant \inf _{c \in\left[y_{3}\right]_{\rho}\left[y_{2}\right]_{\rho}\left[y_{3}\right]_{\rho}} v_{\mathcal{P}}(c)=\inf _{c \in\left[y_{3}\right]_{\rho}} v_{\mathcal{P}}(c)=\overline{v_{\mathcal{P}}}\left(y_{3}\right) .
\end{aligned}
$$

This implies that $\overline{\mu_{\mathcal{P}}}\left(\left[y_{1} y_{2} y_{3}\right]\right) \geqslant \overline{\mu_{\mathcal{P}}}\left(y_{3}\right)$ and $\overline{v_{\mathcal{P}}}\left(\left[y_{1} y_{2} y_{3}\right]\right) \leqslant \overline{v_{\mathcal{P}}}\left(y_{3}\right)$. Then $\overline{A p p}(\mathcal{P})$ is a Pythagorean fuzzy left ideal of T. The proofs of other cases are similar.

(2) Let $y_{1}, y_{2}, y_{3} \in T$.

$$
\begin{aligned}
\underline{\mu_{\mathcal{P}}}\left(\left[y_{1} y_{2} y_{3}\right]\right)=\inf _{y \in\left[y_{1} y_{2} y_{3}\right]_{\rho}} \mu_{\mathcal{P}}(y) & =\inf _{y \in\left[y_{1}\right]_{\rho}\left[y_{2}\right]_{\rho}\left[y_{3}\right]_{\rho}} \mu_{\mathcal{P}}(y) \\
& =\inf _{[a b c] \in\left[y_{1}\right]_{\rho}\left[y_{2}\right]_{\rho}\left[y_{3}\right]_{\rho}} \mu_{\mathcal{P}}([a b c]) \\
& \geqslant \inf _{[a b c] \in\left[y_{1}\right]_{\rho}\left[y_{2}\right]_{\rho}\left[y_{3}\right]_{\rho}} \mu_{\mathcal{P}}(c)=\inf _{c \in\left[y_{3}\right]} \mu_{\mathcal{P}}(c)=\underline{\mu_{\mathcal{P}}}\left(y_{3}\right)
\end{aligned}
$$

and

$$
\begin{aligned}
\underline{v_{\mathcal{P}}}\left(\left[y_{1} y_{2} y_{3}\right]\right)=\sup _{y \in\left[y_{1} y_{2} y_{3}\right]_{\rho}} v_{\mathcal{P}}(y) & =\sup _{y \in\left[y_{1}\right]_{\rho}\left[y_{2}\right]_{\rho}\left[y_{3}\right]_{\rho}} v_{\mathcal{P}}(y) \\
& =\sup _{[a b c] \in\left[y_{1}\right]_{\rho}\left[y_{2}\right]_{\rho}\left[y_{3}\right]_{\rho}} v_{\mathcal{P}}([a b c]) \\
& \leqslant \sup _{[a b c] \in\left[y_{1}\right]_{\rho}\left[y_{2}\right]_{\rho}\left[y_{3}\right]_{\rho}} v_{\mathcal{P}}(c)=\sup _{c \in\left[y_{3}\right]} v_{\mathcal{P}}(c)=\underline{v_{\mathcal{P}}}\left(y_{3}\right) .
\end{aligned}
$$

This implies that $\underline{\mu_{\mathcal{P}}}\left(\left[y_{1} y_{2} y_{3}\right]\right) \geqslant \underline{\mu_{\mathcal{P}}}\left(y_{3}\right)$ and $\underline{v_{\mathcal{P}}}\left(\left[y_{1} y_{2} y_{3}\right]\right) \leqslant \underline{v_{\mathcal{P}}}\left(y_{3}\right)$. Then $\underline{A p p}(\mathcal{P})$ is a Pythagorean fuzzy left ideal of $\mathrm{T}$. The proofs of other cases are similar.

Corollary 3.13. Let $\rho$ be a congruence relation on a ternary semigroup $\mathrm{T}$ and $\mathcal{P}$ be a Pythagorean fuzzy set of $\mathrm{T}$.

(1) If $\mathcal{P}$ is a Pythagorean fuzzy ideal of $\mathrm{T}$, then $\overline{\operatorname{App}}(\mathcal{P})$ is a Pythagorean fuzzy ideal of $\mathrm{T}$.

(2) If $\rho$ is complete and $\mathcal{P}$ is a Pythagorean fuzzy ideal of $\mathrm{T}$, then $\operatorname{App}(\mathcal{P})$ is a Pythagorean fuzzy ideal of $\mathrm{T}$.

Proof. This follows from Theorem 3.12 .

\section{Conclusion}

In this paper, the notion of rough Pythagorean fuzzy sets in ternary semigroups is studied. The idea of rough Pythagorean fuzzy sets is extended to the lower and upper approximations of Pythagorean fuzzy ideals in ternary semigroups and some important properties related to these notions are presented.

\section{Acknowledgment}

The authors would like to thank the reviewer for valuable comments and suggestions.

This paper was supported by Algebra and Applications Research Unit, Prince of Songkla University.

\section{References}

[1] M. A. Ansari, N. Yaqoob, T-rough ideals in ternary semigroups, Int. J. Pure Appl. Math., 86 (2013), 411-424. 1

[2] R. Chinram, Rough prime ideals and rough fuzzy prime ideals in gamma-semigroups, Commun. Korean Math. Soc., 24 (2009), 341-351. 1

[3] H. Garg, A new generalized Pythagorean fuzzy information aggregation using Einstein operations and its application to decision making, Int. J. Intell. Syst., 31 (2016), 886-920. 1 
[4] H. Garg, Generalized Pythagorean fuzzy geometric interactive aggregation operators using Einstein operations and their application to decision making, J. Exper. Theor. Artif. Intell., 30 (2018), 763-794. 1

[5] A. Hussain, T. Mahmood, M. I. Ali, Rough Pythagorean fuzzy ideals in semigroups, Comput. Appl. Math., 38 (2019), 15 pages. 1

[6] A. Iampan, Some properties of ideal extensions in ternary semigroups, Iran. J. Math. Sci. Inform., 8 (2013), 67-74. 1

[7] C. Jirojkul, R. Chinram, R. Sripakorn, Roughness of quasi-ideals in $\Gamma$-semigroups, JP J. Algebra Number Theory Appl., 12 (2008), 113-120. 1

[8] Y. B. Jun, Roughness of gamma-subsemigroups/ideals in gamma-semigroups, Bull. Korean Math. Soc., 40 (2003), 531-536. 1

[9] N. Kuroki, Fuzzy bi-ideals in semigroups, Comment. Math. Univ. St. Paul., 28 (1980), 17-21. 1

[10] N. Kuroki, On fuzzy ideals and fuzzy bi-ideals in semigroups, Fuzzy Sets and Systems, 5 (1981), 203-215. 1

[11] N. Kuroki, Rough ideals in semigroups, Inform. Sci., 100 (1997), 139-163. 1

[12] D. H. Lehmer, A ternary analogue of abelian groups, Amer. J. Math., 54 (1932), 329-338. 1

[13] J. Loś, On the extending of models (I)*, Fund. Math., 42 (1955), 38-54. 1

[14] S. Naz, S. Ashraf, M. Akram, A Novel Approach to Decision-Making with Pythagorean Fuzzy Information, Mathematics, 6 (2018), 28 pages. 1

[15] Z. Pawlak, Rough sets, Internat. J. Comput. Inform. Sci., 11 (1982), 341-356. 1

[16] X. D. Peng, Y. Yang, Some results for Pythagorean fuzzy sets, Int. J. Intell. Syst., 30 (2015), 1133-1160. 1

[17] R. Prasertpong, M. Siripitukdet, On rough sets induced by fuzzy relations approach in semigroups, Open Math., 16 (2018), 1634-1650. 1

[18] P. Ratanaburee, T. Kaewnoi, R. Chinram, Straddles on ternary semigroups, J. Math. Computer Sci., 19 (2019), $246-250$. 1

[19] A. Rosenfeld, Fuzzy groups, J. Math. Anal. Appl., 35 (1971), 512-517. 1

[20] B. Thongkam, T. Changphas, On two-sided bases of ternary semigroups, Quasigroups Related Systems, 23 (2015), 319-324. 1

[21] R. R. Yager, Pythagorean fuzzy subsets, Proceedings of the Joint IFSA World Congress and NAFIPS Annual Meeting (Edmonton, Canada), 2013 (2013), 57-61. 1, 2.2, 2.7, 2.8

[22] R. R. Yager, A. M. Abbasov, Pythagorean member grades, complex numbers, and decision making, Int. J. Intell. Syst., 28 (2013), 436-452. 1, 2.2, 2.7, 2.8

[23] L. A. Zadeh, Fuzzy set, Information and Control, 8 (1965), 338-353. 1 